\title{
ACE inhibitors in non-diabetic renal disease
}

\author{
Richard J Fluck, Anthony E G Raine
}

The advent of angiotensin converting enzyme (ACE) inhibitors has provided not only a new treatment for hypertension but also a specific treatment for hypertension in renal disease, which carries the promise of lessening the progression of renal disease and the risk of additional side effects. In this review we aim to cover the evidence for a role for ACE inhibition in the slowing or halting of progression of chronic renal disease, together with the possible consequences of the use of these drugs in patients with renovascular hypertension and other forms of renal disease.

\section{Progression of renal disease}

Despite the advent of effective renal replacement therapy, mortality and morbidity remain high in patients undergoing dialysis. Effective treatment to slow or halt the progression of renal failure in patients with mild to moderate chronic uraemia is of prime importance. Such treatment would delay the onset of renal replacement therapy, thereby reducing the incidence of associated complications and the limitations it places on the quality of life. Currently, apart from specific treatments for particular renal diseases, efforts to slow the progression of renal failure have focused on two factors: the control of hypertension and dietary protein restriction. Recent data have suggested that progression of renal disease in humans is not significantly improved by protein restriction, hence, the control of hypertension remains the mainstay of conservative measures. ${ }^{1}$ Evidence is now accumulating that there may be a specific additional role for ACE inhibitors in the treatment of renal disease.

\section{Hypertension in renal disease}

All forms of renal disease are associated with hypertension. ${ }^{2}$ The prevalence of hypertension rises as renal disease progresses, and most $(90 \%)$ patients are hypertensive at the time of end stage renal failure. This high rate of development of high blood pressure partly contributes to the excess mortality in this group of patients, ${ }^{3}$ in particular cardiovascular disease, which accounts for $50 \%$ of deaths in patients with end stage renal failure. ${ }^{4}$

Not only is the incidence of hypertension increased by chronic renal failure but hypertension itself may worsen uraemia and accelerate the progression of the disease. It is the breaking of this reciprocal relation by effective antihypertensive treatment that may slow or halt progression towards end stage renal failure.
Theories of progression of renal disease Widely held theories to account for the mechanism of progression of renal disease have emphasised the importance of glomerular capillary hypertension. ${ }^{5}$ In essential hypertension effective autoregulation of the afferent arteriolar resistance seems to protect the glomerulus from the effects of systemic hypertension, but in chronic renal disease this autoregulatory mechanism fails. As a consequence, progressive glomerular injury is aggravated by increased glomerular capillary pressure, culminating in glomerular sclerosis. ${ }^{6}$ Other theories explaining progressive hypertensive glomerular injury have focused on glomerular hypertrophy. These are based on experiments in animals showing a significant correlation between glomerular size and the degree of glomerular sclerosis. ${ }^{7}$ These findings suggest that glomerular hypertrophy is an adaptive response, compensating for the reduction in renal mass, but which itself in some way predisposes to progressive glomerular injury-that is, the process becomes maladaptive.

These other mechanisms provide at least two possible targets that may be used in attempts to slow the progression of renal disease. Firstly, as the intrarenal vasoconstrictor action of angiotensin II is primarily on the efferent arteriole, ACE may reduce glomerular hypertension by preferential vasodilatation of the efferent arteriole, thereby reducing glomerular pressure. Secondly, angiotensin II may act as a growth factor within the glomerulus, and successful use of ACE inhibition may thereby reduce glomerular hypertrophy.

As hypertension itself is a major promoter of declining renal function, however, it is logical in the first place to attempt to reduce or halt this progression by effective control of blood pressure.

\section{Evidence for role of hypertension in the} progression of renal disease

A retrospective study of 198 patients starting long term dialysis suggested that poor control of diastolic blood pressure was associated with an increased rate of decline in renal function. ${ }^{8}$ A controlled study of 79 Afro-Caribbean patients with moderate to severe chronic renal impairment who were not yet receiving dialysis showed that tight control of diastolic blood pressure could halt the progression of renal disease. In this three year study glomerular filtration rate increased slightly in response to treatment. ${ }^{9}$ A more detailed small scale study of 18 patients suggested that controlling the 
diastolic blood pressure at less than $84 \mathrm{~mm} \mathrm{Hg}$ was beneficial in slowing the progression of renal disease, but further lowering blood pressure below $80 \mathrm{~mm} \mathrm{Hg}$ did not confer any additional benefit. ${ }^{10}$

These and many other studies provide good evidence that control of blood pressure is not only beneficial but mandatory in the treatment of chronic renal impairment. Do ACE inhibitors confer additional benefit above antihypertensive treatments?

\section{Specific role for ACE inhibitors in chronic renal failure?}

ROLE OF ANGIOTENSIN II

In addition to the efferent arteriolar vasoconstriction caused by angiotensin II, there is evidence that it acts as a growth factor in both glomerular and tubulointerstitial tissue, as summarised in table $1 .{ }^{11}$ Given these cellular effects of angiotensin II and their possible detrimental consequences for progressive renal impairment, ACE inhibitors might provide additional benefit over and above their antihypertensive action. In studies using the $5 / 6$ nephrectomy experimental model of chronic renal failure, ACE inhibitors lowered glomerular pressure and decreased glomerular sclerosis to a greater degree than comparable antihypertensive treatment with reserpine, hydralazine, and hydrochlorthiazide. ${ }^{12} \mathrm{~A}$ retrospective uncontrolled study in humans has suggested that $\mathrm{ACE}$ inhibition also slowed the progression of clinical renal failure. ${ }^{13}$

Few studies, however, have compared different antihypertensive treatments directly in human renal disease. Glomerular haemodynamics have been studied in normotensive subjects subjected to treatment with either nifedipine or captopril. ${ }^{14}$ The ACE inhibitor reduced the extent of amino acid induced glomerular hyperfiltration, in contrast to nifedipine, which induced hyperfiltration at rest and allowed maximal hyperfiltration to occur when the amino acid infusion was administered. This is interesting as calcium channel blockers have a preferential vasodilator action on the afferent arteriole. This may lead to glomerular hypertension, despite lowering of systemic blood pressure, by impairing glomerular autoregulation. In keeping with these hypotheses, studies in which treatment was started some weeks after subtotal nephrectomy in rats showed that treatment with calcium antagonists was associated with reduced nephron survival and progressive glomerular sclerosis-in contrast to ACE inhibition, which was protective. ${ }^{15}$

Table 1 In vitro effects of angiotensin II on mesangial and proximal tubular cells ${ }^{\prime \prime}$

\begin{tabular}{lll}
\hline Phenotype & Mesangial cells & Tubular cells \\
\hline Growth effect & Proliferation & Hypertrophy \\
Angiotensin II receptor type & $\mathrm{AT}_{1}$ & $\mathrm{AT}_{1}$ and AT \\
Signal transduction pathway & IP3/DAG/Ca & Intracellular cyclic AMP \\
Oncogene activation & Yes & Yes \\
$\begin{array}{l}\text { Collagen synthesis } \\
\text { Existence of genes induced }\end{array}$ & Preferentially type I & Preferentially type IV \\
by angiotensin II & Unknown & Yes \\
\hline
\end{tabular}

IP3, inositol 1,4,5-triphosphate; DAG, diacylglycerol; $\mathrm{Ca}^{2+}$, intracellular calcium.
PROTEINURIA-SURROGATE MARKER FOR PROGRESSION?

Proteinuria may be a marker for both glomerular hypertension and hyperfiltration and, as a consequence, treatments that reduce proteinuria may be beneficial in renal failure. ${ }^{16}$ Long term administration of angiotensin II may increase proteinuria in normal rats. ${ }^{17} \mathrm{~A}$ large study in animals compared the calcium channel blocker verapamil with enalapril ${ }^{15}$; it showed that proteinuria increased in response to calcium antagonism, and this was associated with a high percentage of glomerular sclerosis. This occurred despite equivalent blood pressure control with verapamil and enalapril; in the group given enalapril proteinuria and glomerulosclerosis were reduced.

Protein excretion is also increased in renovascular disease, a high renin state. ${ }^{18}$ More recently, ACE inhibitors have reduced proteinuria in rats with induced chronic uraemia, ${ }^{19}$ and there have been comparable studies in patients with moderate uraemia due to a variety of renal diseases. ${ }^{20}$ Fractional urinary albumin excretion was significantly reduced in patients with mild, moderate, and severe albuminuria, but without a significant change in serum creatinine concentrations. Both enalapril and captopril are effective in reducing proteinuria in hypertensive patients with renal disease. ${ }^{2122}$ In some studies comparing ACE inhibitors with calcium channel blockers a greater reduction in proteinuria was achieved with ACE inhibitors, ${ }^{22} 23$ although recent prospective studies have failed to confirm this. ${ }^{24}$ In addition, in none of these studies was a difference in rate of progression detected in the different treatment groups.

In summary, there is no doubt that ACE inhibitors are effective in reducing proteinuria. However, it remains unproved whether reduction in proteinuria and the implied beneficial effect on glomerular haemodynamics will lead to a more favourable renal outcome in the long term.

BLOOD PRESSURE INDEPENDENT EFFECTS OF ACE INHIBITORS

It remains a matter of controversy whether the beneficial actions of ACE inhibitors relate solely to their antihypertensive properties or whether additional mechanisms independent of blood pressure control also contribute. Ikeda et al and Bianchi et al found that blood pressure control in the different treatment groups was equivalent. ${ }^{22} 23$ However, small differences, or an unmeasured change in the profile of blood pressure control over 24 hours, may well result in significant changes in renal outcome. The possibility that ACE inhibitors may have an additional beneficial effect through reducing the effect of angiotensin II as a growth factor is of current interest. Actions of ACE inhibitors independent of those on blood pressure may potentially be investigated through their use in normotensive patients with renal disease, with subsequent assessment of their effects on progression of renal disease and on proteinuria. Such studies will 
Table 2 Risk factors for renal artery stenosis ${ }^{32}$

Risk factor

Old age

Old age

Peripheral vascular disease Congestive cardiac failure Raised serum creatinin History of smoking Female sex require long follow up times and large numbers of patients; no data are yet available.

A small study of ACE inhibition in 14 patients with chronic renal failure and proteinuria greater than $1 \mathrm{~g} /$ day included five normotensive subjects. ${ }^{25}$ Overall, creatinine clearance and urine protein excretion both improved during a 30 month follow up period, though no comparisons were made between normotensive and hypertensive subjects. In a crossover study subjects given conventional triple drug treatment were given captopril for 12 months. ${ }^{26}$ This small study of 10 patients suggested that the ACE inhibitor halted the decline of renal failure with no change in control of blood pressure. Of interest in this study was the fact that blood pressure control was identical and urinary protein excretion did not improve during the 12 months of captopril treatment. Despite this, the progression of renal disease, as assessed by reciprocal plots of creatinine concentration, was retarded. A criticism of this study, as of many others, is that renal function was poorly assessed, with failure to use definitive methods such as isotopic assessment of glomerular filtration rate.

\section{Summary}

In non-diabetic chronic renal failure there is at present incomplete evidence that ACE inhibitors have an additional benefit in the slowing of progressive renal disease over and above their antihypertensive properties. In theory, their beneficial action might result both from decreased glomerular hypertension, through selective dilatation of the afferent arteriole, and from blocking the actions of angiotensin II as a growth factor in both the glomerulus and the interstitium. The evidence is, however, flawed, incomplete, and in many cases circumstantial. Long term placebo controlled crossover studies are still required to answer these questions.

ACE inhibition in renovascular disease The widespread use of ACE inhibitors in clinical practice requires an awareness of their side effects, particularly those related to underlying renal disease. The main area of concern is the use of these drugs in occult or overt renovascular disorders. This awareness must encompass not only renal physicians but all physicians who prescribe ACE inhibitors. The dilemma arises because ACE inhibitors are very effective in reducing blood pressure in patients with renovascular hypertension, most of whom have an activated renin-angiotensin system. However, the possible long term adverse effects of ACE inhibitors on renal function in unilateral stenosis of the kidney are worrying; they may precipitate severe renal impairment in bilateral renovascular disease.

Incidence of renovascular disease

The incidence of renovascular disease is difficult to ascertain as the condition is largely painless and asymptomatic. If only one kidney is stenosed, there will be no warning change in the indicators of renal function measured routinely. For these reasons, a high index of suspicion must be maintained in patients at risk.

The proportion of patients with end stage renal failure who have renovascular disease as the primary diagnosis ranges from $8 \%$ to $14 \% .{ }^{27} 28$ This incidence increases with age at time of presentation. Renovascular disease may be a relevant factor in up to $16 \%$ of patients with acute presentation of renal failure. ${ }^{29}$ Mailloux et al reported that the prognosis in patients with end stage renal failure due to renovascular disease was worse than that in patients with diabetic nephropathy. ${ }^{30}$

The comparatively high rate of renovascular disease in patients with known renal disease gives no information about its incidence in the general population. Nevertheless, data from general angiography indicate that renovascular disease is common. Patients investigated for peripheral vascular disease with angiography had significant renal artery stenosis in $42 \%$ of cases, ${ }^{31}$ and $19 \%$ of 1303 patients undergoing cardiac catheterisation had renal artery stenosis. $^{32}$ The predictors for renal artery stenosis included increased age, the presence of peripheral vascular disease, raised serum creatinine concentrations, smoking, and female sex (table 2). Another study found that half of the 450 patients undergoing angiography for peripheral vascular disease had an occlusion of a renal artery. ${ }^{33}$ It is of note that a fifth of these were in fact normotensive at the time of study.

ACE inhibitors may acutely impair renal function in patients with renal artery stenosis, and this was first described in a renal transplant recipient over 10 years ago. ${ }^{34}$ In patients with hypertension due to renovascular disease plasma renin concentration is usually high. Treatment with an ACE inhibitor generally produces a gratifying fall in blood pressure, but this may be accompanied by the risk of an appreciable deterioration in renal function. This risk arises particularly in patients with a single functioning kidney or with bilateral renal artery stenosis. ${ }^{35}$

MECHANISM

The mechanism of acute renal failure accompanying the use of ACE inhibitors in renovascular disease is still imperfectly understood. The generally accepted explanation is that arterial stenosis results in impaired perfusion of the afferent arteriolar circulation. The increased renin secretion this induces causes a compensatory efferent arteriolar vasoconstriction, which maintains glomerular hydraulic pressure and thus maintains a normal glomerular filtration rate. ${ }^{36}$ When an ACE inhibitor is administered the renin-angiotensin cascade is inhibited, with a decrease in angiotensin II concentration and therefore a loss of the efferent arteriolar compensatory vasoconstriction. This leads to a reduction in glomerular capillary pressure, and glomerular filtration and acute renal failure may result. 
Cases of acute renal failure are often multifactorial, and renal failure associated with ACE inhibitors in renovascular disease is no exception. Cases may be exacerbated in patients who are given non-steroidal antiinflammatory drugs at the same time, ${ }^{37}$ who are salt depleted, particularly when receiving loop diuretics, ${ }^{38}$ or who have other underlying renal parenchymal disease. Hence a continuing awareness of patients at risk, especially those with volume depletion, diabetes mellitus, or underlying chronic renal disease, is required.

\section{CLINICAL COURSE}

In general, acute renal failure secondary to the use of ACE inhibitors in renovascular disease should be reversible, but this is not always the case. Firstly, the condition must be recognised early enough to allow the treatment to be withdrawn in time. Secondly, blood pressure and circulatory volume should be restored to minimise the risk of long term renal ischaemic damage or thrombosis.

Acute renal failure due to ACE inhibitors in renovascular disease is symptomless, clinical sequelae often becoming apparent only when the patient has established acute renal failure. Therefore it is recommended that patients at risk have their renal function assessed before treatment is started and within 2-5 days after the first dose. In addition, the starting dose should be at the lower end of the recommended range for any given ACE inhibitor.

\section{Acute renal failure in non-renovascular conditions}

In addition to concern over ACE inhibitors in renovascular disease, acute renal failure associated with their use in other renal disorders is not unknown. The use of enalapril has been associated with reversible renal failure in hypertensive nephrosclerosis in which the main additional risk factor was a local heat wave, perhaps associated with volume depletion. ${ }^{39}$ All cases were reversible and were successfully managed by dose reduction or withdrawal of the ACE inhibitor. A series of five patients with autosomal dominant polycystic kidney disease were reported to have suffered acute renal failure associated with the use of ACE inhibitors. ${ }^{40}$ Again, there were other precipitating factors, including intravascular volume depletion secondary to diuretic treatment in at least two cases. In two of the patients studied acute renal failure occurred again after rechallenge with the ACE inhibitor. These patients were extensively investigated and no evidence of renal artery stenosis was found.

\section{Use of ACE inhibitors in renal transplantation}

Hypertension is a common accompaniment of cadaveric renal transplantation, and its incidence and prevalence have increased with the introduction of cyclosporin. ${ }^{41}$ Consequently, patients receiving renal transplants are increasingly exposed to the use of ACE inhibitors for the treatment of hypertension. In such patients who have one functioning kidney the incidence of renal artery stenosis is significant. ${ }^{42}$ There is no doubt that the use of ACE inhibitors should be discouraged in the early stages of renal transplantation, while renal function is being established. Treatment with ACE inhibitors for resistant hypertension following transplantation should be preceded by investigation for occult renal artery stenosis, particularly as this may be the cause of the hypertension.

In view of the potential benefit of ACE inhibitors in progressive renal disease, it is natural to speculate whether these drugs may have a beneficial action in slowing or preventing the decline in renal function that results from long term graft rejection. As yet there are no long term placebo controlled trials available to assess this question, but several studies have assessed proteinuria in renal transplant recipients. Use of lisinopril in a group of 12 hypertensive recipients with proteinuria but no evidence of renal artery stenosis resulted in a decrease in proteinuria from 2.98 (SD 2.06) g/24 h to $2.06(2.9) \mathrm{g} / 24 \mathrm{~h}^{43}$ A control group of patients with comparable blood pressure control but who were not taking ACE inhibitors did not show any changes in the degree of proteinuria. The study lasted only three months and glomerular filtration rate remained unchanged, but the short term nature of the study allows no conclusions about the progression of renal dysfunction.

\section{ACE inhibitors in end stage renal failure}

The pharmacokinetics of ACE inhibitors in renal failure have been extensively reviewed. ${ }^{44}$ The absorption of ACE inhibitors is generally unchanged in renal disease, but after metabolism in the liver most active compounds are excreted by the kidney. As a consequence, recommended maximal doses of these drugs are reduced in patients with renal failure, with the exception of fosinopril, whose clearance is primarily hepatic. In maintenance haemodialysis most ACE inhibitors, including captopril, enalapril, and lisinopril, have high clearances. By contrast, quinapril, which has high plasma protein binding, has an extremely low clearance of $0.6 \mathrm{ml} / \mathrm{min}$.

A recent problem reported with ACE inhibitor treatment in patients receiving haemodialysis is the development of anaphylactic reactions associated with the use of AN69 polyacrylnitrile high flux dialysers. The mechanism is largely unknown, but it may be a result of an increase in bradykinin synthesis induced by an interaction of blood and blood compounds with the polymer and a concomitant blockade of bradykinin metabolism because of inhibition of kininase by the ACE inhibitor. ${ }^{45}$ The current recommendation is that ACE inhibitors should not be used in association with these particular high flux dialysers. 
In patients receiving continuous ambulatory peritoneal dialysis the pharmacokinetic data on ACE inhibitors are few. Quinapril excretion in the peritoneal dialysate seems to be negligible compared with that of captopril, where a single dose led to the detection of captopril in the dialysate. ${ }^{44}$

\section{ACE inhibitors as a diagnostic tool}

The use of captopril renography has enjoyed recent popularity. In essence, the technique entails isotopic renal scanning before and after a single dose of captopril ( $25 \mathrm{mg}$ orally). ${ }^{46} 47$ After the administration of captopril the expectation is a delay in tracer handling on the side of the renal artery stenosis. Other data suggest that the specificity and sensitivity of this test for the absolute diagnosis of renovascular disease are poor, but that the results are a better predictor of successful outcome of revascularisation or angioplasty. ${ }^{48}$

\section{Summary}

In non-diabetic renal disease ACE inhibitors have brought both benefit and problems. On the one hand, they are undoubtedly effective antihypertensive agents and data suggest that they may have a beneficial role in the prevention of progression of renal disease, although further placebo controlled double blind trials of adequate duration are required. On the other hand, their widespread use in elderly patients and those with generalised atherosclerosis has increased the risk of acute renal failure when occult renovascular disease is present. Awareness among physicians of the high rate of renovascular disease in populations at risk is to be encouraged.

1 Levey AS, Bosch JP, Coggins CH, et al. Effects of diet and blood pressure on creatinine clearance $\left(\mathrm{C}_{C R}\right)$ and serum
creatinine $\left(\mathrm{P}_{C R}\right)$ in the MDRD study (abstract). fournal of the American Society of Nephrology 1993;4:253.

2 Brown MA, Whitworth JA. Hypertension in human renal disease. $\mathcal{F}$ Hypertens 1992;10:701-12.

$3 \mathrm{Ma} \mathrm{KW}$, Greene EL, Raij L. Cardiovascular risk factors in chronic renal failure and hemodialysis populations. $A m \mathcal{J}$ Kidney Dis 1992;6:505-13.

4 Raine AEG, Margreiter R, Brunner FP, et al. Report on the management of renal failure in Europe. XXII 1991. Nephrol Dial Transplant 1992;7(suppl 2):7-35.

5 Brenner BM, Meyer TW, Hostetter TH. Dietary protein intake and the progressive nature of kidney disease: the role of hemodynamically mediated glomerular injury in the pathogenesis of progressive glomerular sclerosis in aging, renal ablation, and intrinsic renal disease. $N$ Engl Med 1982;307:652-9.

6 Tolins JP, Raij. Antihypertensive therapy and the progression of chronic renal disease. Are there renoprotective drugs? Semin Nephrol 1991:11:538-48.

7 Yoshida Y, Fogo A, Sharaga H, et al. Serial micropuncture analysis of single nephron function in the rat model of subtotal renal ablation. Kidney Int 1988;33:855-67.

8 Brazy PC, Stead W, Fitzwilliam JF. Progression of renal insufficiency: role of blood pressure. Kidney Int 1989;35:670-4.

9 Pettinger WA, Lee HC, Reisch J, Mitchell HC. Long-term improvement in renal function after short-term blood pressure control in hypertensive nephrosclerosis. Hypertension 1989;13:766-72.

10 Wight JP, Brown CB, El Nahas AM. Effect of control of hypertension on

11 Wolf G, Neilson EG. Angiotensin II as a renal growth factor. Fournal of the American Society of Nephrology 1993;3:1531-40.

12 Anderson S, Rennke HG, Brenner BM. Therapeutic advantages of converting enzyme inhibitors in arresting progressive renal disease associated with hypertension. $\mathscr{f}$ Clin Invest 1986;77:1993-2000.
13 Mann JFE, Reisch C, Ritz E. Use of angiotensin-converting enzyme inhibitors for the preservation of kidney function. A retrospective study. Nephron 1990;55(suppl 1):38-42.

14 Bohler J, Woitas R, Keller E, Reetze-Bonorden P, Schollmeyer PJ. Effect of nifedipine and captopril on glomerular hyperfiltration in normotensive man. $A m \mathfrak{f}$ Kidney 1992;20:132-9.

15 Brunner FP, Thiel G, Hermle M, Bock A, Mihatsch MJ. Long-term enalapril and verapamil in rats with reduced renal mass. Kidney Int 1989;36:969-77.

16 Glassock RJ. Focus on proteinuria. Am $f$ Nephrol 1990;10(suppl 1):88-93

17 Bohrer MP, Deen WM, Robertson CR, et al. Mechanism of angiotensin II induced proteinuria in the rat. $A m \mathcal{F}$ Physiol 1977;233:F13-21.

18 Pasternack A, Eklund J, Krohn K. Renal artery stenosis and the nephrotic syndrome. Acta Med Scand 1967; 181:265-8.

19 Anderson S, Meyer TW, Rennke HG, Brenner BM. Control of glomerular hypertension limits glomerular injury in rats with reduced renal mass. $\mathcal{f}$ Clin Invest 1985;76:612-9.

20 Kurkus J, Thysell H. Reduction of albuminuria after angiotensin converting enzyme inhibition of various renal disorders. Scand f Urol Nephrol 1990;24:63-8.

21 Grazi G, Cirami C, Panichi V, et al. Renal effects of enalapril in hypertensive patients with glomerulonephritis. Nephrol Dial Transplant 1989;4:396-8.

22 Ikeda T, Nakayama D, Gomi T, Sakurai T, Yamazaki T, Yuhara M. Captopril, an angiotensin I-converting Yuhara $M$. Captopril, an angiotensin l-converting patients with renal diseases. Nephron 1989;52:72-5.

23 Bianchi S, Bigazzi R, Baldari G, Campese VM. Long-term effects of enalapril and nicardipine on urinary albumin effects of enalapril and nicardipine on urinary albumin excretion in patients with chronic renal insuffici

24 Zucchelli P, Zuccala A, Borghi M, et al. Long term comparison between captopril and nifedipine in the progression of renal insufficiency. Kidney Int 1992 42:452-8.

25 Ferder LF, Inserra F, Daccordi H, Romano L, Fernandez A, Tessler J. Effects of enalapril on renal parameters in patients with primary glomerulopathies associated with chronic renal failure. Drugs 1990;30(suppl 2):40-6.

26 Ruilope LM, Miranda B, Morales JM, Rodico JL, Romera JC, Raij L. Converting enzyme inhibition in chronic renal failure. Am $\mathcal{F}$ Kidney Dis 1989;13:120-6.

27 Broyer M, Donckerwolcke RA, Brunner FP, et al. Combined report on regular dialysis and transplantation in Europe. Proceedings of the European Dialysis and Transplant Association 1983;20:2-75.

28 Scoble JE, Maher ER, Hamilton G, Dick R, Sweny P, Moorhead JF. Atherosclerotic renovascular disease causing renal impairment-a case for treatment. Clin Nephrol 1989;31:119-22.

29 Kalra PA, Mamtora H, Holmes AM, Waldek S. Renovascular disease and complications of angiotensin
converting enzyme inhibitor therapy. $Q \mathcal{7}$ Med converting enzym

30 Mailloux LU, Belluci AG, Mossey RT, et al. Predictors of survival in patients undergoing dialysis. $\mathrm{Am} \mathcal{F} \mathrm{Med}$ $1988 ; 84: 855-62$

31 Choudri AH, Cleland JGF, Rowlands PC, Tran TL, McCarthy M, Al-Kutoubi MA. Unsuspected renal artery stenosis in peripheral vascular disease. $B M F$ 1990;301:1197-8.

32 Harding MB, Smith LR, Himmelstein SI, et al. Renal artery stenosis: prevalence and associated risk factors in patients undergoing routine cardiac catheterization. fournal of the American Society of Nephrology 1992; 2:1608-16.

33 Swartbol P, Thorvinger BO, Parsson H, Norgen L. Renal artery stenosis in patients with peripheral vascular disease and its correlation with hypertension. A retrospective study. Int Angiology 1992;11:195-9.

34 Farrow PR, Wilkinson R. Reversible renal failure during treatment with captopril. BMF 1979; i: 1680 .

35 Miyamori J, Yasuhara S, Takeda Y, et al. Effects of converting enzyme inhibition on split renal function in converting enzyme inhibition on split renal function in

36 Hricik DE, Brown PJ, Kopelman R, Goorno WE, Madias NE, Dzau VJ. Captopril induced functional renal insufficiency in patients with bilateral renal artery stenoses or renal artery stenosis in a single kidney. $N$ Engl Med 1983;308:373-6.

37 Seelig CB, Maloley PA, Campbell JR. Nephrotoxicity associated with concomitant ACE inhibitor and NSAID therapy. South Med F 1990;83:1144-8.

38 Lee H-C, Pettinger WA. Diuretics potentiate the angiotensin converting enzyme inhibitor associated acute renal dysfunction. Clin Nephrol 1992;38:236-7.

39 Toto RD, Mitchell HC, Lee H-C, Milam C, Pettinger WA. Reversible renal insufficiency due to angiotensin converting enzyme inhibitors in hypertensive nephrosclerosis. Ann Intern Med 1991;115:513-9.

40 Chapman AB, Gabow PA, Schrier RW. Reversible renal failure associated with angiotensin converting enzyme inhibitors in polycystic kidney disease. Ann Intern Med 1991;115:769-73.

41 Luke RG. Hypertension in renal transplant recipients. Kidney Int 1987;31:1024-37.

42 Curtis JJ, Luke RG, Whelchel JD, Diethelm AG, Jones P, Dustan HP. Inhibition of angiotensin converting enzyme in renal transplant recipients with hypertension. $N$ Engl f Med 1983;308:377-81. 
43 Traindl O, Falger S, Reading S, et al. The effects of lisinopril on renal function in proteinuric renal transplant recipients. Transplantation 1993;55:1309-13.

44 Hoyer J, Schulte K-L, Lenz T. Clinical pharmacokinetics of angiotensin converting enzyme (ACE) inhibitors in renal failure. Clin Pharmacokinet 1993;24:230-54.

45 Tielemans C, Madhoun P, Lenaers M, et al. Anaphylactoid reactions during hemodialysis on AN69 membranes in patients receiving ACE inhibitors. Kidney Int 1990; 38:982-4.
46 Nally JV Jr. Renal physiology of renal artery stenosis. Implications for captopril stimulated renography. $A m \mathcal{F}$ Implications for captopril

47 de Zeeuv WD, Jonker GJ, Hovinga TK, et al. The mechanism and diagnostic value of angiotensin I converting enzyme inhibition renography. Am $\mathcal{f}$ Hypertens 1991;4:741-4S.

48 Fommei E, Mezzasalma L, Ghione S, et al. European captopril radionuclide test multicenter study. Preliminary results. Am f Hypertens 1991;4:690-7S. 